\title{
Drug-induced prevention of gastrectomy- and ovariectomy-induced osteopaenia in the young female rat
}

\author{
N Andersson ${ }^{1,2}$, V V Surve ${ }^{1}$, D Lehto-Axtelius ${ }^{3}$, C Ohlsson $^{4}$, \\ R Håkanson ${ }^{1}$, K Andersson ${ }^{2}$ and B Ryberg ${ }^{2}$ \\ ${ }^{1}$ Department of Pharmacology, Institute of Physiological Sciences, Lund University, BMC F-13, S-221 84 Lund, Sweden \\ ${ }^{2}$ AstraZeneca Research and Development, Mölndal, Sweden \\ ${ }^{3}$ Department of Radiology, Centre for Oral Health Sciences, Malmö University, Malmö, Sweden \\ ${ }^{4}$ Department of Internal Medicine, Research Centre for Endocrinology and Metabolism, Sahlgrenska University Hospital, Göteborg, Sweden \\ (Requests for offprints should be addressed to R Håkanson; Email: Rolf.Hakanson@farm.lu.se) \\ ( $\mathrm{N}$ Andersson and $\mathrm{V} \vee$ Surve contributed equally to this work)
}

\begin{abstract}
Both ovariectomy (Ovx) and gastrectomy (Gx) induce osteopaenia in rats and humans. While the effect of Ovx has been ascribed to oestrogen deficiency, the underlying mechanism behind Gx is poorly understood. Alendronate, oestrogen and parathyroid hormone (PTH) are known to prevent the osteopaenia induced by Ovx in rats. The purpose of the present study was to determine whether alendronate, oestrogen or PTH could also prevent Gxevoked osteopaenia. Rats were Ovx-, Gx-, or were sham-operated (Sham) and were then treated with alendronate $(50 \mu \mathrm{g} / \mathrm{kg} /$ day $)$, oestrogen $(10 \mu \mathrm{g} / \mathrm{kg} /$ day $)$ or $\mathrm{PTH}(1-84)(75 \mu \mathrm{g} / \mathrm{kg} /$ day $)$ for eight weeks. At sacrifice, serum PTH was unaffected by surgery (Ovx, $64 \pm 8 \mathrm{pg}$ / $\mathrm{ml}$; Gx, $75 \pm 13$ pg/ml; Sham, $58 \pm 11 \mathrm{pg} / \mathrm{ml})$. The bone mineral density (BMD) of the fifth lumbar vertebra (L5) was analysed. Ovx and Gx reduced the BMD (ash weight/volume) of the L5 by $15 \pm 4 \%$ and $22 \pm 3 \%$ respectively. Trabecular BMD and the cortical bone mineral content (BMC) of the femur were assessed using
\end{abstract}

peripheral computed tomography. Both Ovx and Gx markedly reduced trabecular BMD in the metaphyseal area of the distal femur (Ovx, $-37 \pm 7 \%$; Gx, $-49 \pm 7 \%)$. The cortical BMC of the femur was only slightly reduced. Alendronate prevented trabecular bone loss after both Ovx and Gx, while oestrogen and PTH prevented trabecular bone loss after Ovx but not after Gx.

In conclusion, the bisphosphonate alendronate prevented both $\mathrm{Ovx}$ - and Gx-induced trabecular bone loss. In contrast, $\mathrm{PTH}$ and oestrogen prevented Ovx-induced but not Gx-induced trabecular bone loss, suggesting that the mechanism behind the trabecular bone loss in Ovx rats differs from that in Gx rats. The results support the notion that the mechanism of action for the bone-sparing effect of these drugs differs. The ability of alendronate, and probably also other bisphosphonates, to prevent Gx-evoked osteopaenia in the rat might be of potential clinical interest when dealing with post-Gx osteopaenia in humans.

Journal of Endocrinology (2002) 175, 695-703

\section{Introduction}

The ovariectomized (Ovx) rat is an established animal model for testing ways to prevent and treat postmenopausal osteoporosis (Wronski et al. 1985, Yamazaki \& Yamaguchi 1989, Kalu 1991, Thompson et al. 1995). Oestrogen deficiency results in increased bone turnover with excess bone resorption and significant loss of trabecular bone (Bagi et al. 1997). In cortical bone, an increased bone turnover rate results in endocortical resorption. However, this is compensated by accelerated periosteal growth and the net cortical bone loss is small or non-existent (Turner et al. 1987, Bain et al. 1993).

Gastrectomy $(\mathrm{Gx})$, i.e. resection of the glandular part of the stomach, also leads to osteopaenia in the rat (Klinge et al. 1995, Rümenapf et al. 1997, Lehto-Axtelius et al. 1998, Mühlbauer et al. 1998, Gepp et al. 2000, Stenström et al. 2000, Surve et al. 2001a,b). In humans, gastrectomy results in osteopaenia via mechanisms that are poorly understood due to difficulties in performing clinical studies which differentiate between many putative factors acting simultaneously (Tovey et al. 1991, 1992, Melton et. al. 1999, Heiskanen et al. 2001). However, in the rat studies have been performed which rule out calcium malabsorption (Persson et al. 1993, Rümenapf et al.1997, LehtoAxtelius et al. 2002, Surve et al. 2002), vitamin D deficiency (Axelson et al. 1991, Rümenapf et al. 1997, 1998), loss of gastric acid (Persson et al. 1993) and secondary hyperparathyroidism (Rümenapf et al. 1997, 1998, Mühlbauer et al. 1998, Wojtyczka et al. 1998) 


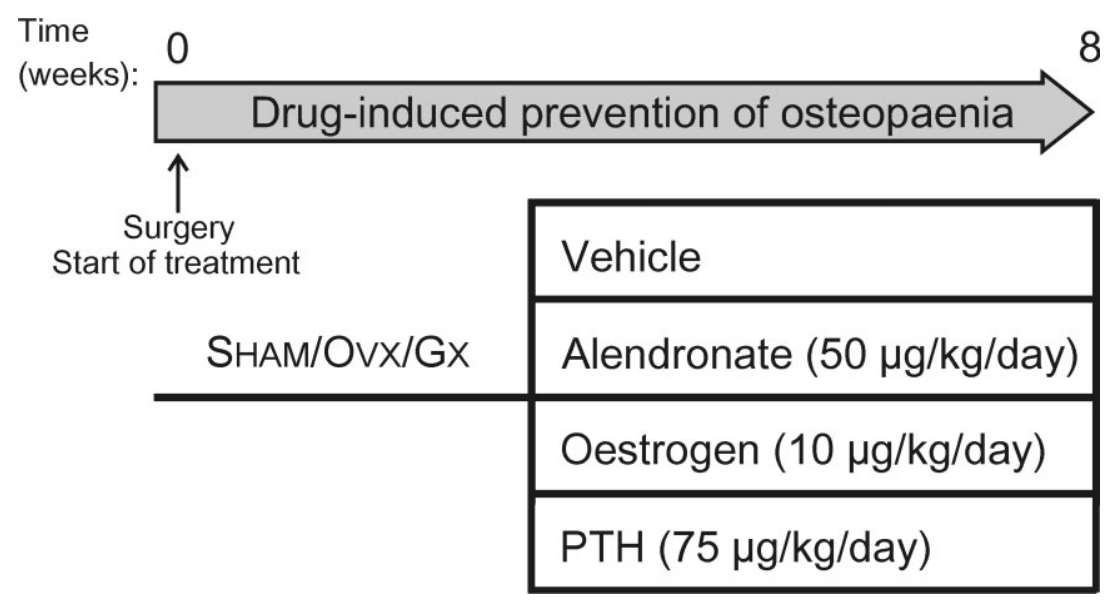

Figure 1 Experimental design. Rats were subjected to sham operation (Sham), ovariectomy $(\mathrm{Ovx})$, or gastrectomy $(\mathrm{Gx})$. The three groups (32 rats in each) were subdivided into four preventive treatment groups (vehicle, alendronate, oestrogen and PTH, 8 rats in each). The drugs were administered on a daily basis for eight weeks, starting on the day after surgery.

as being the major causes of Gx-evoked bone loss. Gx-evoked osteopaenia differs in some respects from that induced by Ovx (Surve et al. 2001a,b), suggesting that the underlying mechanisms are different. Both Ovx and Gx affect trabecular bone and at times also cortical bone. In addition, however, $\mathrm{Gx}$ induces a quite spectacular effect in the calvaria of the rat (Klinge et al. 1995, Lehto-Axtelius et al. 1998, Mühlbauer et al. 1998, Surve et al. 2001a,b).

Osteoporotic patients are currently treated with oestrogen or bisphosphonates. Oestrogen replacement is the most common treatment of post-menopausal osteoporosis (Cosman \& Lindsay 1999). Bisphosphonates, such as alendronate, reduce fracture risk because they increase bone mass by reducing bone turnover (Ravn et al. 1996, Cummings et al. 1998, Fisher et al. 1999). Parathyroid hormone $(\mathrm{PTH})$ is an anabolic hormone, which has attracted attention recently as a potential remedy for osteoporosis. Animal studies (Mitlak et al. 1996, Sato et al. 1997) as well as clinical studies (Rosen \& Rackoff 2001) have shown that PTH, when administered intermittently, exerts an anabolic effect on bone. The anabolic action of PTH is most evident in trabecular bone while cortical bone mass is either unaffected or only moderately increased. However, if PTH is administered continuously it has a catabolic effect on bone (Dempster et al. 1993, 1995).

In the present study, we assessed the ability of alendronate, oestrogen, and PTH to prevent osteopaenia in the rat following Gx or Ovx. The purpose of the study was to explore whether the osteopaenia induced by Gx and Ovx responded similarly to preventive treatment with any of these drugs. Bones were removed and examined after 8 weeks of preventive drug treatment.

\section{Materials and Methods}

\section{Animals}

Ninety-six female, three-month-old Sprague-Dawley rats were obtained from M\&B, Skensved, Denmark. The rats were allowed an acclimatisation period of seven days prior to the start of the study. The animals were maintained in groups of three in Macrolon cages, on a $12 \mathrm{~h}$ light $/ 12 \mathrm{~h}$ darkness cycle with access to standard rat food pellets (1.0\% calcium and $0.7 \%$ phosphorus, Lactamin, Vadstena, Sweden) and tap water available ad libitum. Body weights were determined at the beginning of the study and weekly thereafter. In order to prevent anaemia, $\mathrm{Gx}$ rats were injected with $400 \mu \mathrm{g} / \mathrm{kg}$ vitamin $\mathrm{B}_{12}$ (Betolvex, Dumex, Copenhagen, Denmark) and $20 \mathrm{mg} / \mathrm{kg}$ iron $\left(\mathrm{Fe}^{3+}\right)$ sorbitol (Jectofer, AstraZeneca, Södertälje, Sweden) by the intramuscular route once every second week (beginning the first week after surgery). The experiments had been approved by the local animal welfare committee before the study started.

\section{Experimental design}

The rats were randomly assigned to one of three groups and then subjected to various types of surgery (Fig. 1): gastrectomy ( $\mathrm{Gx}, n=32)$, ovariectomy (Ovx, $n=32)$, and sham-operation (Sham, $n=32$ ). The three groups were subdivided into four treatment groups (8 rats in each). Drug treatment started the day after surgery. The drugs were administered on a daily basis for eight weeks. The treatment groups were: Sham+vehicle (sesame oil), subcutaneous injection (s.c. inj.), Sham $+50 \mu \mathrm{g} / \mathrm{kg} /$ day 
alendronate (corresponding to an effective daily dose of $10 \mu \mathrm{g}$ compound phosphorus per kg; alendronate hydrochloride was synthesised at AstraZeneca R\&D, Mölndal, Sweden), s.c. inj., Sham $+10 \mu \mathrm{g} / \mathrm{kg} /$ day oestrogen (oestradiol-3-benzoate, Boehringer Ingelheim, Germany), s.c. inj., Sham $+75 \mu \mathrm{g} / \mathrm{kg} /$ day PTH (human recombinant PTH(1-84), Allelix Biopharmaceuticals, Mississauga, ON, Canada), s.c. inj., Ovx+vehicle, Ovx+alendronate, Ovx + oestrogen, Ovx $+\mathrm{PTH}, \mathrm{Gx}+$ vehicle, Gx+alendronate, $\mathrm{Gx}+$ oestrogen, and $\mathrm{Gx}+\mathrm{PTH}$. Citrate buffered saline (10 mM, pH 5.5) was used as vehicle for PTH. Saline was used as vehicle for alendronate. The doses chosen have been shown to be effective in preventing Ovx-induced osteopaenia (Wronski et al. 1988, Seedor et al. 1991, Shen et al. 1995).

The rats were killed under isoflurane (Forene, Abbot, Abbot Park, IL, USA) anaesthesia by exsanguination (cardiac puncture). Blood was drawn and serum was stored at $-20{ }^{\circ} \mathrm{C}$ until analysis (for gastrin and osteocalcin). Uteri were removed, and wet weights were recorded. Femurs, tibiae, and the fifth lumbar vertebrae (L5) were dissected out and cleaned of soft tissue. Each L5 was wrapped in saline-soaked gauze and stored at $-20{ }^{\circ} \mathrm{C}$ until determination of bone density. The left femurs and tibiae were fixed in $10 \%$ formalin for four days at $4{ }^{\circ} \mathrm{C}$, washed thoroughly thereafter and stored in $70 \%$ ethanol at $4{ }^{\circ} \mathrm{C}$.

\section{Surgery}

Before surgery, the rats were anaesthetised with an intraperitoneal injection of ketamine hydrochloride (Ketalar, Parke-Davis, Barcelona, Spain) and xylazine (Rompun, Bayer, Leverkusen, Germany) at doses of 90 and $4.5 \mathrm{mg} /$ $\mathrm{kg}$ respectively. Gx was performed by resection of the glandular part of the stomach and anastomosing the rumen to the duodenum (Lehto-Axtelius et al. 1998). Successful Gx was verified by the demonstration of reduced concentrations of gastrin in serum. Ovx and sham operations were performed using an abdominal approach (mid-line abdominal incision). Successful Ovx was verified by demonstrating reduced uterine weight (data not shown). There was no mortality in the Ovx or Sham groups. In the Gx group the mortality was about $10 \%$.

\section{Measurement of serum gastrin, osteocalcin and PTH}

Determination of serum gastrin was performed as previously described (Stadil \& Refeld 1973). Rat gastrin-17 was used as standard. The concentration of gastrin in serum was expressed as pmol equivalents of rat gastrin-17 per litre. Serum osteocalcin was measured using a commercially available enzyme-linked immunosorbent assay (Rat-MID Osteocalcin ELISA, Osteometer BioTech, Herlev, Denmark). The concentration of osteocalcin was expressed as ng equivalents of rat osteocalcin per ml. Serum PTH was measured using a commercially available immunoradiometric assay (Rat PTH IRMA, Immunotopics Inc., San Clemente, CA, USA). The concentration of $\mathrm{PTH}$ in serum was expressed as $\mathrm{pg}$ equivalents of rat $\mathrm{PTH}(1-34)$ per ml.

\section{Measurement of bone mineral density}

The volume of the vertebral body of L5 (with the two epiphyseal ends, the posterior pedicle arch, and the spinous process removed, height $\sim 4 \mathrm{~mm}$ ) was determined by Archimedes' principle. The vertebrae were subsequently incinerated at $600{ }^{\circ} \mathrm{C}$ for $12 \mathrm{~h}$; the resulting ash was weighed after cooling in a desiccator. The ash weight divided by the volume gave the bone mineral density $\left(\mathrm{BMD}, \mathrm{mg} / \mathrm{cm}^{3}\right)$.

\section{Peripheral quantitative computed tomography ( $p Q C T$ )}

Computerised tomography was performed with the Stratec pQCT XCT Research M (Norland Corp., Fort Atkinson, WI, USA) specifically modified for use on small bone specimens (software version 5.4B; operating at $70 \mu \mathrm{m}$ resolution) (Rosen et al. 1995, Windahl et al. 1999, Andersson et al. 2001). The machine was calibrated with a standard of hydroxyapatite embedded in acrylic plastic. pQCT was used to analyse cross-sections of the distal metaphysis and mid-diaphysis of the left femur. During the measurements the excised femurs were placed in a test tube filled with $70 \%$ ethanol. Metaphyseal scans were performed to measure the trabecular BMD $\left(\mathrm{mg} / \mathrm{cm}^{3}\right)$. The scout view of the pQCT system was used to locate the growth plate. The metaphyseal scan line was positioned $2.5 \mathrm{~mm}$ proximal to the distal growth plate. This area of the femoral metaphysis is rich in trabecular bone. The trabecular bone region was defined as the inner 45\% of the scanned bone area. Cortical BMD $\left(\mathrm{mg} / \mathrm{mm}^{3}\right)$ was determined in a $0.1 \mathrm{~mm}$ cross-section of the middiaphysis. This scan was also used to determine geometrical parameters such as the cortical thickness $(\mathrm{mm})$, the cortical cross sectional area $\left(\mathrm{mm}^{2}\right)$, the periosteal circumference $(\mathrm{mm})$, the endocortical circumference $(\mathrm{mm})$ and the cortical bone mineral content (BMC, $\mathrm{mg} / \mathrm{mm}$ ). The inter-assay coefficient of variation for the pQCT measurements was less than $2 \%$. The lengths of the femur and tibiae were also determined at the same time.

\section{Statistical analysis}

The results are presented as means \pm S.E.M. The effects of drugs or the effects of surgery were analysed by one-way analysis of variance (ANOVA); $P<0 \cdot 05$ was considered statistically significant. Whenever statistically significant differences were found between the various experimental 
Table 1 Effects of the three drugs on the body weight gain. Results are means \pm S.E.M. $(n=7-8$ rats per group)

\begin{tabular}{|c|c|c|c|c|}
\hline & Vehicle & Alendronate & Oestrogen & PTH \\
\hline \multicolumn{5}{|l|}{ Sham-operated rats } \\
\hline Starting weight (g) & $225 \pm 5$ & $229 \pm 5$ & $226 \pm 4$ & $226 \pm 4$ \\
\hline Final weight (g) & $294 \pm 6$ & $288 \pm 10$ & $296 \pm 7$ & $291 \pm 8$ \\
\hline Change in weight (\%) & $31 \pm 2$ & $26 \pm 2$ & $31 \pm 4$ & $29 \pm 4$ \\
\hline \multicolumn{5}{|l|}{ Ovx rats } \\
\hline Starting weight (g) & $213 \pm 5$ & $213 \pm 5$ & $209 \pm 6$ & $211 \pm 6$ \\
\hline Final weight $(\mathrm{g})$ & $347 \pm 12^{\#}$ & $336 \pm 16^{\#}$ & $274 \pm 6^{*}$ & $364 \pm 16^{\#}$ \\
\hline Change in weight (\%) & $63 \pm 2^{\#}$ & $58 \pm 4^{\#}$ & $31 \pm 4^{*}$ & $73 \pm 5^{\#}$ \\
\hline \multicolumn{5}{|l|}{ Gx rats } \\
\hline Starting weight (g) & $234 \pm 2$ & $231 \pm 5$ & $231 \pm 3$ & $231 \pm 4$ \\
\hline Final weight $(\mathrm{g})$ & $287 \pm 7$ & $276 \pm 15$ & $273 \pm 8$ & $288 \pm 5$ \\
\hline Change in weight (\%) & $23 \pm 3$ & $19 \pm 1$ & $18 \pm 4$ & $25 \pm 2$ \\
\hline
\end{tabular}

Statistical significance was assessed for the differences between the sham-operated rats and each of the two surgery groups or between the vehicle-treated groups and each of the three drug-treated groups.

${ }^{\#} P<0.05$ versus sham; ${ }^{*} P<0.05$ versus vehicle (ANOVA).

Table 2 Effects of the three drugs on length $(\mathrm{mm})$ of tibia and femur in Gx, Ovx and Sham rats. Results are means \pm S.E.M. ( $n=7-8$ rats per group)

\begin{tabular}{|c|c|c|c|c|}
\hline & Vehicle & Alendronate & Oestrogen & PTH \\
\hline \multicolumn{5}{|l|}{ Sham } \\
\hline Tibia & $39 \cdot 7 \pm 0 \cdot 3$ & $38 \cdot 3 \pm 0 \cdot 4^{*}$ & $39 \cdot 5 \pm 0 \cdot 4$ & $39 \cdot 1 \pm 0 \cdot 3$ \\
\hline Femur & $35 \cdot 0 \pm 0 \cdot 2$ & $34 \cdot 5 \pm 0 \cdot 2$ & $34 \cdot 7 \pm 0 \cdot 2$ & $34 \cdot 9 \pm 0 \cdot 1$ \\
\hline \multicolumn{5}{|l|}{ Ovx } \\
\hline Tibia & $39 \cdot 9 \pm 0 \cdot 3$ & $38 \cdot 4 \pm 0 \cdot 3^{*}$ & $38 \cdot 8 \pm 0 \cdot 2^{*}$ & $39 \cdot 7 \pm 0 \cdot 4$ \\
\hline Femur & $35 \cdot 6 \pm 0 \cdot 4$ & $34 \cdot 8 \pm 0 \cdot 4$ & $34 \cdot 4 \pm 0 \cdot 3$ & $35 \cdot 7 \pm 0.5$ \\
\hline \multicolumn{5}{|l|}{ Gx } \\
\hline Tibia & $39 \cdot 1 \pm 0 \cdot 2$ & $38 \cdot 2 \pm 0 \cdot 4$ & $38 \cdot 8 \pm 0 \cdot 4$ & $39 \cdot 7 \pm 0 \cdot 2$ \\
\hline Femur & $35 \cdot 4 \pm 0 \cdot 3$ & $34 \cdot 7 \pm 0 \cdot 2$ & $34 \cdot 7 \pm 0 \cdot 3$ & $35 \cdot 5 \pm 0 \cdot 3$ \\
\hline
\end{tabular}

Statistical significance was assessed for the differences between the vehicle-treated groups and each of the three drug-treated groups. ${ }^{*} P<0 \cdot 05$ (ANOVA).

groups by ANOVA, individual differences were assessed by post hoc analysis (Dunnett's test).

\section{Results}

Body weights and skeletal dimensions of bones

Ovx rats gained weight more rapidly than Gx or shamoperated rats (Table 1). Oestrogen treatment reduced the body weight gain of Ovx rats but not that of Gx and Sham rats. Neither PTH nor alendronate treatment affected the final body weights in any of the groups.

The lengths of the tibia and femur were not adversely affected by surgery. The length of the tibia was reduced by alendronate treatment in Sham and Ovx rats and was slightly reduced by oestrogen in Ovx rats. No effects on the lengths of the tibia or femur were noted in the other groups (Table 2).
$B M D$

The BMD (ash weight/volume) of L5 was determined. Ovx and Gx reduced the BMD by $15 \pm 4 \%(P<0 \cdot 05)$ and $22 \pm 3 \%(P<0 \cdot 05)$ respectively (Fig. $2 A)$. Compared with vehicle treatment, alendronate raised the BMD of $\mathrm{L} 5$ in both Ovx $(34 \pm 5 \%, P<0 \cdot 05)$ and $\mathrm{Gx}(21 \pm 3 \%, P<0 \cdot 05)$ rats, but not in sham-operated rats. No effects of oestrogen were seen in any of the groups. PTH significantly increased the BMD in the L5 of Ovx and sham-operated, but not of Gx rats $(P<0 \cdot 05)$.

L5 contains both trabecular and cortical bone. In order to determine whether the main effects of surgery and of the preventive drug treatments were on trabecular or cortical bone, the trabecular BMD in the metaphysis and the cortical bone in the mid-diaphysis of the femur were measured using pQCT. Eight weeks after surgery both Ovx and Gx rats had a significantly reduced trabecular $\mathrm{BMD}$ in the distal femur (Ovx, $-37 \pm 7 \%, \quad P<0 \cdot 05$; 
A

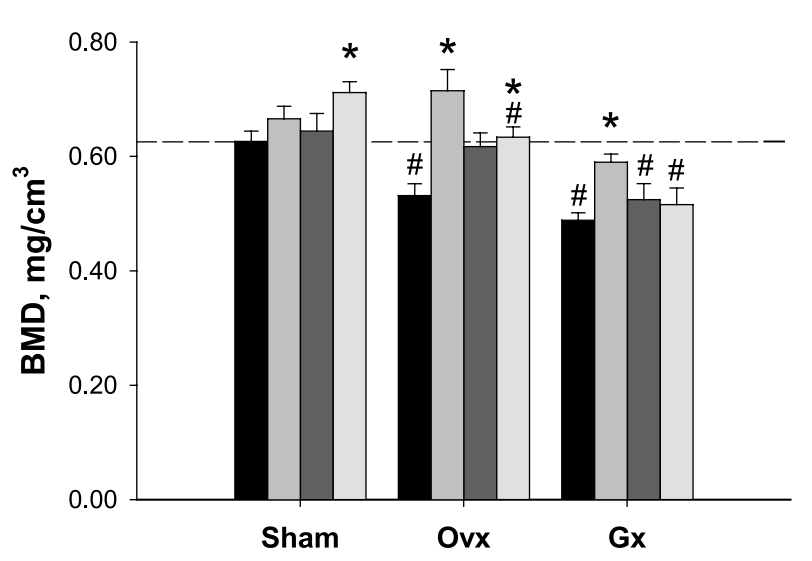

B

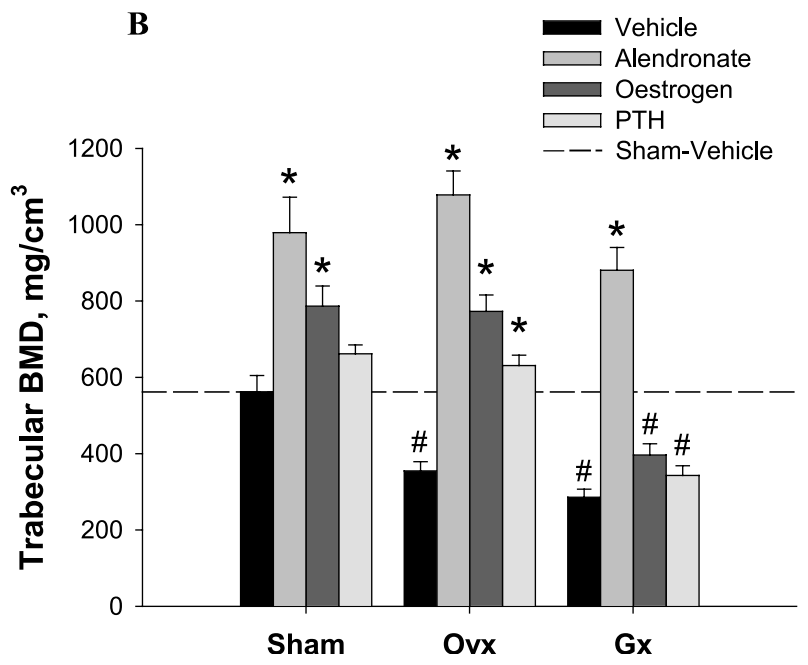

Figure 2 (A) Bone mineral density (BMD) of the fifth lumbar vertebral body (ash weight/volume). (B) Trabecular BMD in the metaphysis of the femur (measured by pQCT). The trabecular bone region was defined as the inner $45 \%$ of the scanned bone area. Results are means \pm S.E.M. ( $n=7-8$ rats per group).

Vehicle-treated rats were compared with the various drug-treated groups and sham-operated rats were compared with the two surgery groups. ${ }^{*} P<0 \cdot 05$ versus vehicle; ${ }^{\#} P<0.05$ versus Sham (ANOVA).

Gx, $-49 \pm 7 \%, P<0 \cdot 05$ ) (Fig. 2B). Alendronate modified the loss of trabecular BMD in all three surgery groups, while PTH and oestrogen prevented the trabecular osteopaenia induced by Ovx but not that induced by Gx.

In contrast to trabecular bone, cortical bone responded poorly to both Gx and Ovx as well as to the preventive drug treatment (Table 3). However, the cortical thickness was reduced - albeit moderately - in all Gx groups compared with Sham, while Ovx seemed to be without effect on cortical bone. Oestrogen-treated Ovx

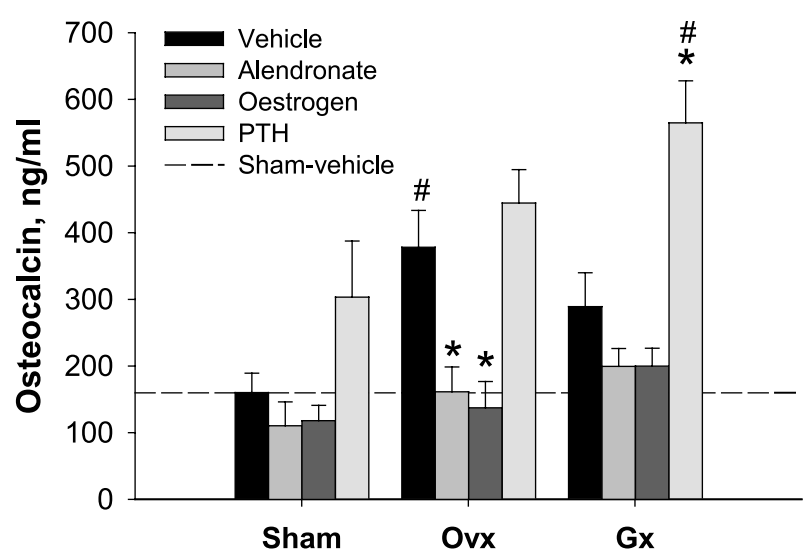

Figure 3 Effects of alendronate, oestrogen and PTH on the serum osteocalcin concentration at sacrifice. Results are means \pm S.E.M. ( $n=7-8$ rats per group). Sham-operated rats were compared with each of the two surgery groups and vehicle-treated rats were compared with each of the three drug-treated groups. ${ }^{*} P<0 \cdot 05$ versus vehicle; ${ }^{\#} P<0 \cdot 05$ versus Sham (ANOVA).

rats showed a reduced periosteal and endocortical circumference compared with oestrogen-treated sham-operated rats.

\section{Serum gastrin, osteocalcin and PTH}

The serum concentration of gastrin was low in all Gx groups at sacrifice while it was normal in all other groups (Gx, $23 \pm 1 \mathrm{pmol} / \mathrm{l}$; Ovx, $74 \pm 4 \mathrm{pmol} / \mathrm{l}$; Sham, $72 \pm 4 \mathrm{pmol} / 1(P<0 \cdot 05$ ANOVA $))$. The serum concentration of osteocalcin, a marker of bone formation/ turnover, was increased in Ovx rats but not in Gx rats (Fig. 3). Alendronate and oestrogen treatment suppressed the serum osteocalcin concentration in the Ovx rats to Sham levels. The serum concentration of PTH at sacrifice was unchanged in all groups (Gx, $75 \pm 13 \mathrm{pg} / \mathrm{ml}$; Ovx, $64 \pm 8 \mathrm{pg} / \mathrm{ml}$; Sham, $58 \pm 11 \mathrm{pg} / \mathrm{ml}$ (not significant, ANOVA)).

\section{Discussion}

Ovx and Gx cause osteopaenia in both rats and humans. Although both Ovx-evoked and Gx-evoked osteopaenia reflect decreased formation and accelerated degradation and turnover of bone, the pathogenetic mechanisms behind the bone loss seem to differ (Surve et al. 2001a,b). Gx reduces the percentage of bone in the calvaria and lowers the trabecular and cortical bone content. Ovx affects trabecular bone (and only affects cortical bone to a minor extent) but not calvarial bone (Surve et al. 2001b). Today, bisphosphonates and oestrogen replacement are used clinically to treat osteoporosis and soon PTH will be available as an additional treatment option. In this study we examine whether alendronate, oestrogen or $\mathrm{PTH}$ will 
Table 3 Effects of drug treatment on cortical bone parameters measured in the mid-diaphysis of the femur by pQCT. Results are means \pm S.E.M. $(n=7-8$ rats per group)

\begin{tabular}{|c|c|c|c|c|}
\hline & Vehicle & Alendronate & Oestrogen & PTH \\
\hline \multicolumn{5}{|l|}{ Sham-operated rats } \\
\hline Cortical BMC (mg/mm) & $7 \cdot 53 \pm 0 \cdot 15$ & $7 \cdot 55 \pm 0 \cdot 20$ & $7 \cdot 53 \pm 0 \cdot 15$ & $7 \cdot 83 \pm 0 \cdot 20$ \\
\hline Cortical BMD (mg/mm³) & $1 \cdot 356 \pm 0 \cdot 004$ & $1 \cdot 364 \pm 0 \cdot 003$ & $1 \cdot 348 \pm 0 \cdot 002$ & $1 \cdot 358 \pm 0 \cdot 007$ \\
\hline Cortical area $\left(\mathrm{mm}^{2}\right)$ & $5 \cdot 56 \pm 0 \cdot 12$ & $5 \cdot 54 \pm 0 \cdot 15$ & $5 \cdot 59 \pm 0 \cdot 11$ & $5 \cdot 77 \pm 0 \cdot 14$ \\
\hline Cortical thickness (mm) & $0 \cdot 62 \pm 0.01$ & $0.62 \pm 0.01$ & $0 \cdot 60 \pm 0 \cdot 01$ & $0.65 \pm 0.01$ \\
\hline Periosteal circumference $(\mathrm{mm})$ & $10 \cdot 96 \pm 0 \cdot 13$ & $10 \cdot 85 \pm 0 \cdot 18$ & $11 \cdot 19 \pm 0 \cdot 11$ & $10 \cdot 87 \pm 0.09$ \\
\hline Endocortical circumference (mm) & $7 \cdot 10 \pm 0 \cdot 11$ & $6 \cdot 94 \pm 0 \cdot 20$ & $7 \cdot 41 \pm 0 \cdot 10$ & $6 \cdot 77 \pm 0 \cdot 09$ \\
\hline \multicolumn{5}{|l|}{ Ovx rats } \\
\hline Cortical BMC (mg/mm) & $7 \cdot 41 \pm 0 \cdot 13$ & $7 \cdot 87 \pm 0 \cdot 33$ & $7 \cdot 11 \pm 0 \cdot 16$ & $7 \cdot 81 \pm 0 \cdot 27$ \\
\hline Cortical BMD (mg/mm $\left.\mathrm{mm}^{3}\right)$ & $1 \cdot 353 \pm 0.004$ & $1 \cdot 351 \pm 0.006$ & $1 \cdot 355 \pm 0 \cdot 004$ & $1 \cdot 332 \pm 0.005^{\star \#}$ \\
\hline Cortical area $\left(\mathrm{mm}^{2}\right)$ & $5 \cdot 48 \pm 0.09$ & $5 \cdot 83 \pm 0 \cdot 26$ & $5 \cdot 25 \pm 0 \cdot 11$ & $5 \cdot 86 \pm 0 \cdot 19$ \\
\hline Cortical thickness (mm) & $0 \cdot 60 \pm 0.01$ & $0.62 \pm 0.01$ & $0 \cdot 60 \pm 0.01$ & $0.65 \pm 0.01^{*}$ \\
\hline Periosteal circumference (mm) & $10 \cdot 99 \pm 0 \cdot 14$ & $11 \cdot 33 \pm 0 \cdot 31$ & $10 \cdot 62 \pm 0 \cdot 13^{\#}$ & $11 \cdot 08 \pm 0 \cdot 21$ \\
\hline Endocortical circumference (mm) & $7 \cdot 20 \pm 0 \cdot 18$ & $7 \cdot 45 \pm 0 \cdot 26$ & $6 \cdot 85 \pm 0 \cdot 14^{\#}$ & $7 \cdot 02 \pm 0 \cdot 20$ \\
\hline \multicolumn{5}{|l|}{ Gx rats } \\
\hline Cortical BMC (mg/mm) & $7 \cdot 24 \pm 0 \cdot 10$ & $7 \cdot 23 \pm 0 \cdot 07$ & $6 \cdot 84 \pm 0 \cdot 13^{\#}$ & $7 \cdot 18 \pm 0 \cdot 14$ \\
\hline Cortical BMD $\left(\mathrm{mg} / \mathrm{mm}^{3}\right)$ & $1 \cdot 353 \pm 0.005$ & $1 \cdot 345 \pm 0 \cdot 004^{\#}$ & $1 \cdot 357 \pm 0 \cdot 008$ & $1 \cdot 340 \pm 0 \cdot 005$ \\
\hline Cortical area $\left(\mathrm{mm}^{2}\right)$ & $5 \cdot 36 \pm 0.09$ & $5 \cdot 38 \pm 0.06$ & $5 \cdot 04 \pm 0 \cdot 09^{\#}$ & $5 \cdot 36 \pm 0 \cdot 12$ \\
\hline Cortical thickness (mm) & $0 \cdot 58 \pm 0 \cdot 01^{\#}$ & $0 \cdot 57 \pm 0 \cdot 01^{\#}$ & $0.57 \pm 0.01^{\#}$ & $0 \cdot 58 \pm 0 \cdot 01^{\#}$ \\
\hline Periosteal circumference (mm) & $11 \cdot 01 \pm 0 \cdot 18$ & $11 \cdot 17 \pm 0 \cdot 10$ & $10 \cdot 69 \pm 0 \cdot 19^{\#}$ & $11 \cdot 05 \pm 0 \cdot 15$ \\
\hline Endocortical circumference (mm) & $7 \cdot 34 \pm 0 \cdot 20$ & $7 \cdot 56 \pm 0 \cdot 12$ & $7 \cdot 13 \pm 0 \cdot 23$ & $7 \cdot 39 \pm 0 \cdot 16^{\#}$ \\
\hline
\end{tabular}

Statistical significance was assessed for the differences between the sham-operated rats and each of the two surgery groups ( $P<0 \cdot 05$ ) or between the vehicle-treated groups and each of the three drug-treated groups $\left({ }^{*} P<0 \cdot 05\right)$ (ANOVA).

prevent the osteopaenia induced by Ovx or Gx in rats. This is the first study in which these three drugs have been used in an attempt to prevent Gx-evoked osteopaenia. Our results confirm that alendronate and oestrogen are able to prevent the trabecular bone loss induced by Ovx (Wronski et al. 1988, Seedor et al. 1991). In addition, we show that PTH at a dose previously shown to be effective in the treatment of an established Ovx-evoked osteopaenia (Shen et al. 1995) also prevented Ovx-induced bone loss. In contrast, $\mathrm{Gx}$-induced bone loss could be prevented by alendronate but not by PTH or oestrogen. Thus, the evidence suggests that the mechanism behind the Ovxevoked osteopaenia in rats differs from that induced by Gx.

Both Ovx and Gx were found to induce a significant decrease in the BMD of L5. The vertebral body is rich in trabecular bone but contains also significant amounts of cortical bone. In order to determine whether the main effects of Ovx and Gx were on trabecular or cortical bone we measured the trabecular BMD in the metaphysis of the distal femur using pQCT. Cortical bone was measured in the mid-diaphysis of the same femur. The trabecular BMD was reduced following Ovx and Gx while the cortical BMC was unaffected, indicating that Ovx as well as Gx primarily affect trabecular bone. However, in the Gx group a reduced cortical thickness was observed. This was not the case in the Ovx group. These results are in accordance with those that have been reported previously (Wronski et al. 1985, 1988, Surve et al. 2001a,b).

Ovx was associated with an increased body weight, which was prevented by treatment with oestrogen, con- firming the results of several earlier studies (Yamazaki \& Yamaguchi 1989, Kalu 1991, Kalu et al. 1991). The effects of oestrogen on body weight and uterine weights (data not shown) demonstrate that a functional dose of oestrogen was given. This dose of oestrogen reversed the Ovxinduced trabecular bone loss but did not prevent the Gx-induced osteopaenia. This finding indicates that the trabecular bone loss following $\mathrm{Gx}$ is initiated and driven by a mechanism other than that causing Ovx-evoked osteopaenia.

Alendronate prevented both Ovx-induced and Gxinduced trabecular bone loss while no effect was seen on cortical bone. The exact mechanism of action for alendronate, as well as other bisphosphonates, is not well understood. The antiresorptive effect of bisphosphonates impairs the final steps in bone remodelling, directly interfering with the activated osteoclasts. Once internalised within the osteoclasts, it is thought to interfere with the mevalonate-to-cholesterol pathway (Bergstrom et al. 2000). The reduced length of the tibia observed in this study has been described previously with a similar dose of alendronate (Seedor et al. 1991). The fact that alendronate prevented the $\mathrm{Gx}$-evoked trabecular bone loss supports the view that calcium malabsorption is not responsible for the bone loss (Lehto-Axtelius et al. 2002, Surve et al. 2002).

Intermittent injections of $\mathrm{PTH}$ result in an anabolic effect on bone in both Sham and Ovx rats (Dempster et al. 1993, 1995). Although most studies of the in vivo effects of PTH have been performed on Ovx rats with established osteopaenia, PTH has been given at different dosages to 
intact or sham-operated rats (4-7 weeks old) for relatively short periods of time (6-24 days) (Hock et al. 1988, Gunness-Hey \& Hock 1989, Hock \& Gera 1992, Uzawa et al. 1995, Frolik et al. 1999). These studies have shown an increase in the trabecular bone volume (measured by histomorphometry) of $15-100 \%$. In a fairly recent study by Frolik et al. (1999), 4-week-old male rats were treated with $\mathrm{PTH}(1-34)$ (equivalent of $100 \mu \mathrm{g} / \mathrm{kg} /$ day $\mathrm{PTH}(1-$ 84)) for 4 weeks. As a result, the BMD in the metaphysis of the proximal tibia was increased by $52 \%$. In the present study we treated 3-month-old female rats with PTH for 8 weeks. The increase in trabecular BMD in the metaphysis of the distal femur following PTH treatment of sham-operated rats was not statistically significant. Interestingly, PTH prevented the Ovx-evoked but not the Gx-evoked bone loss. The results of the present study confirm earlier observations that intermittent PTH treatment prevents Ovx-induced trabecular bone loss (Dempster et al. 1993, 1995). However, as seen for oestrogen, the anabolic/protective effect of PTH seen in the Ovx rats was not apparent in the Gx rats, supporting the notion that the mechanism behind Gx-induced osteopaenia differs from that behind Ovx-induced osteopaenia.

Biochemical markers of bone turnover, such as serum osteocalcin, are widely used clinically to monitor the metabolic activity of the bone (Woitge \& Seibel 2001). Our study showed elevated serum osteocalcin concentrations 8 weeks after Ovx, and this elevation was suppressed by alendronate or oestrogen. These findings are consistent with previous results (Shen et al. 1995, Frolik et al. 1996). Although previous studies (Shen et al. 1993, 1995, Meng et al. 1996) have reported increased osteocalcin levels following PTH treatment in Ovx rats, this study failed to show statistical significance. However, PTH prevented the Ovx-induced bone loss. On the other hand, PTH raised the serum osteocalcin concentration in the Gx rats, suggesting an increased rate of bone formation (Woitge \& Seibel 2001). However, this was not associated with an altered bone mass. Again, the mechanism behind Gx-induced osteopaenia seems to differ from that behind Ovx-induced osteopaenia.

There is a frequently voiced suspicion that nutritional deficiencies cause or at least contribute to the Gx-evoked osteopaenia. This suspicion is triggered by earlier observations that the normal body weight gain is slowed down by Gx (see e.g. Klinge et al. 1995). However, it is unlikely that either the Gx-induced or the Ovx-induced osteopaenia is a result of generalised nutritional deficiency, as in this study neither the Gx group nor the Ovx group were growth retarded (see also Surve et al. 2001a,b). A specific deficiency of calcium is also unlikely because previous reports have described a lack of effect of calcium supplementation (Persson et al. 1993, Klinge et al. 1995, Lehto-Axtelius et al. 1998, 2002) and because the serum PTH concentration was unaffected by surgery (this study). Vitamin D deficiency is known to cause impaired absorp- tion and osteomalacia (Imawari et al. 1980). This is unlikely to be the cause of the Gx-evoked osteopaenia since the serum concentration of 1,25-dihydroxyvitamin $\mathrm{D}_{3}$ is raised following Gx (Axelson et al. 1991, Rümenapf et al. 1998). Elevated levels of 1,25-dihydroxyvitamin $D_{3}$ could be caused by secondary hyperparathyroidism. However, there was no evidence of hyperparathyroidism in this study confirming several observations made earlier (Mühlbauer et al. 1998, Rümenapf et al. 1998, Wojtyczka et al. 1998). It may be argued that removal of the acid-producing part of the stomach may cause acidosis with consequences for calcium/bone metabolism. However, complete inhibition of gastric acid secretion by proton pump inhibitors does not cause osteopaenia (Persson et al. 1993). Hence, the results support the view that the stomach is important for bone metabolism through mechanisms that are unrelated to dietary deficiencies or lack of gastric acid.

In conclusion, PTH and oestrogen prevented Ovxinduced but not Gx-induced trabecular bone loss, suggesting that the mechanism behind the trabecular bone loss in $\mathrm{Gx}$ rats differs from that in Ovx rats. In contrast, the bisphosphonate alendronate prevented both $\mathrm{Gx}$ - and Ovx-induced trabecular bone loss. The preventive effect of alendronate but not of oestrogen or PTH on Gxinduced bone loss supports the notion that the mechanism of action for the bone-sparing effect of these substances differs. The ability of alendronate, and probably also other bisphosphonates, to prevent $\mathrm{Gx}$-evoked osteopaenia in the rat, might be of potential clinical interest when dealing with post-Gx osteopaenia in humans.

\section{Acknowledgements}

The authors are grateful to Ms Susanne Arvidsson, Ms Agneta Karlsson, Ms Dorota Kakol-Palm, Ms Anna Themner-Persson and Ms Maud Pettersson for their expert technical assistance. We would also like to thank the SWEGENE Centre for Bio-Imaging (CBI) at Göteborg University for technical support regarding Image analysis. This study was supported by AstraZeneca, the Swedish Medical Research Council (K2001-72X-04250-28A and 04x-1007), the Swedish Foundation for Strategic Research, the Lundberg Foundation, the Swedish Medical Society, the Novo Nordisk Foundation, the Torsten and Ragnar Söderbergs Foundation, the Emil and Vera Cornell Foundation, the Petrus and Augusta Hedlunds Foundation, the Swedish Association Against Rheumatic Disease, the A Påhlsson Foundation, the Crafoord Foundation and the Medical Faculty, Lund University.

\section{References}

Andersson N, Lindberg MK, Ohlsson C, Andersson K \& Ryberg B 2001 Repeated in vivo determinations of bone mineral density 
during parathyroid hormone treatment in ovariectomized mice. Journal of Endocrinology 170 529-537.

Axelson J, Persson P, Gagnemo-Persson R \& Håkanson R 1991 Importance of the stomach in maintaining calcium homoeostasis in the rat. Gut 32 1298-1302.

Bagi CM, Ammann P, Rizzoli R \& Miller SC 1997 Effect of estrogen deficiency on cancellous and cortical bone structure and strength of the femoral neck in rats. Calcified Tissue International 61 336-344.

Bain SD, Jensen E, Celino DL, Bailey MC, Lantry MM \& Edwards MW 1993 High-dose gestagens modulate bone resorption and formation and enhance estrogen-induced endosteal bone formation in the ovariectomized mouse. Journal of Bone and Mineral Research $\mathbf{8}$ 219-230.

Bergstrom JD, Bostedor RG, Masarachia PJ, Reszka AA \& Rodan G 2000 Alendronate is a specific, nanomolar inhibitor of farnesyl diphosphate synthase. Archives of Biochemistry and Biophysics $\mathbf{3 7 3}$ 231-241.

Cosman F \& Lindsay R 1999 Selective estrogen receptor modulators: clinical spectrum. Endocrine Reviews 20 418-434.

Cummings SR, Black DM, Thompson DE, Applegate WB, Barrett-Connor E, Musliner TA, Palermo L, Prineas R, Rubin SM, Scott JC, Vogt T, Wallace R, Yates AJ \& LaCroix AZ 1998 Effect of alendronate on risk of fracture in women with low bone density but without vertebral fractures: results from the Fracture Intervention Trial. Journal of the American Medical Association 280 2077-2082.

Dempster DW, Cosman F, Parisien M, Shen V \& Lindsay R 1993 Anabolic actions of parathyroid hormone on bone. Endocrine Reviews 14 690-709.

Dempster DW, Cosman F, Parisien M, Shen V \& Lindsay R 1995 Anabolic actions of parathyroid hormone on bone: update 1995. In Endocrine Reviews Monographs, vol. 4, pp 247-250. Ed. A Negro-Vilar. Baltimore, USA: The Endocrine Society Press.

Fisher JE, Rogers MJ, Halasy JM, Luckman SP, Hughes DE, Masarachia PJ, Wesolowski G, Russel RG, Rodan GA \& Reszka AA 1999 Alendronate mechanism of action: geranylgreanol, an intermediate in the mevalone pathway, prevents inhibition of osteoclast formation, bone resorption, and kinase activation in vitro. PNAS 96 133-138.

Frolik CA, Bryant HU, Black EC, Magee DE \& Chandrasekhar S 1996 Time-dependent changes in biochemical bone markers and serum cholesterol in ovariectomized rats: effects of raloxifene $\mathrm{HCl}$, tamoxifen, estrogen, and alendronate. Bone 18 621-627.

Frolik CA, Cain RL, Sato M, Harvey AK, Chandrasekhar S, Black EC, Tashjian AH Jr \& Hock JM 1999 Comparison of recombinant human PTH(1-34) (LY333334) with a C-terminally substituted analog of human PTH-related protein(1-34) (RS-66271): in vitro activity and in vivo pharmacological effects in rats. Journal of Bone and Mineral Research 14 163-172.

Gepp H, Koch M, Schwille PO, Erben RG, Rümenapf G, Schmiedl A \& Fries W 2000 Vagus-sparing gastric fundectomy in the rat: development of osteopaenia, relationship to urinary phosphate and net acid secretion, serum gastrin and vitamin D. Research in Experimental Medicine 200 1-16.

Gunness-Hey M \& Hock JM 1989 Loss of the anabolic effect of parathyroid hormone on bone after discontinuation of hormone in rats. Bone $10447-452$.

Heiskanen JT, Kröger H, Pääkkönen MT, Parviainen MT, Lamberg-Allardt C \& Alhava E 2001 Bone mineral density after total gastrectomy. Bone 28 123-127.

Hock J M \& Gera I 1992 Effects of continuous and intermittent administration and inhibition of resorption on the anabolic response of bone to parathyroid hormone. Journal of Bone and Mineral Research 7 65-72.

Hock JM, Gera I, Fonseca J \& Raisz LG 1988 Human parathyroid hormone-(1-34) increases bone mass in ovariectomized and orchidectomized rats. Endocrinology 122 2899-2904.
Imawari M, Kozawa K, Akanuma Y, Koizumi S, Itakura H \& Kosaka K 1980 Serum 25-hydroxy-vitamin D and vitamin D-binding protein levels and mineral metabolism after partial and total gastrectomy. Gastroenterology 79 255-258.

Kalu DN 1991 The ovariectomized rat model of postmenopausal bone loss. Bone and Mineral 15 175-191.

Kalu DN, Salerno E, Liu CC, Echon R, Ray M, Garza-Zapata M \& Hollis BW 1991 A comparative study of the actions of tamoxifen, estrogen and progesterone in the ovariectomized rat. Bone and Mineral 15 109-123.

Klinge B, Lehto-Axtelius D, Åkerman M \& Håkanson R 1995 Structure of calvaria after gastrectomy. An experimental study in the rat. Scandinavian Journal of Gastroenterology 30 952-957.

Lehto-Axtelius D, Stenström M \& Johnell O 1998 Osteopaenia after gastrectomy, fundectomy or antrectomy: an experimental study in the rat. Regulatory Peptides 78 41-50.

Lehto-Axtelius D, Surve VV, Johnell O \& Håkanson R 2002 Effects of calcium deficiency and calcium supplementation on gastrectomy-induced osteopaenia in the young male rat. Scandinavian Journal of Gastroenterology 37 299-306.

Melton III LJ, Crowson CS, Khosla S \& O’Fallon WM 1999 Fracture risk after surgery for peptic ulcer disease: a population-based cohort study. Bone 25 61-67.

Meng XW, Liang XG, Birchman R, Wu DD, Dempster DW, Lindsay R \& Shen V 1996 Temporal expression of the anabolic action of PTH in cancellous bone of ovariectomized rats. Journal of Bone and Mineral Research 11 421-429.

Mitlak BH, Burdettemiller P, Schoenfeld D \& Neer RM 1996 Sequential effects of chronic human PTH (1-84) treatment of estrogen-deficiency osteopaenia in the rat. Journal of Bone and Mineral Research 11 430-439.

Mühlbauer RC, Schenk RK, Chen D, Lehto-Axtelius D \& Håkanson R 1998 Morphometric analysis of gastrectomy-evoked osteopaenia. Calcified Tissue International 62 323-326.

Persson P, Gagnemo-Persson R, Chen D, Axelson J, Nylander AG, Johnell O \& Håkanson R 1993 Gastrectomy causes bone loss in the rat: is lack of gastric acid responsible? Scandinavian Journal of Gastroenterology 28 301-306.

Ravn P, Clemmesen B, Riis BJ \& Christiansen C 1996 The effect on bone mass and bone markers of different doses of ibandronate - a new bisphosphonate for prevention and treatment of postmenopausal osteoporosis - a 1-year, randomized, double-blind, placebo-controlled dose-finding study. Bone 19 527-533.

Rosen CJ \& Rackoff PJ 2001 Emerging anabolic treatments for osteoporosis. Rheumatic Disease Clinics of North America 27 215-233.

Rosen HN, Tollin S, Balena R, Middlebrooks VL, Beamer WG, Donohue LR, Rosen C, Turner A, Holick M \& Greenspan SL 1995 Differentiating between orchidectomized rats and controls using measurements of trabecular bone density: a comparison among DXA, histomorphometry, and peripheral quantitative computerized tomography. Calcified Tissue International 57 35-39.

Rümenapf G, Schwille PO, Erben RG, Schreiber M, Fries W, Schmiedl A \& Hohenberger W 1997 Osteopaenia following total gastrectomy in the rat - state of mineral metabolism and bone histomorphometry. European Surgical Research 29 209-221.

Rümenapf G, Schwille PO, Erben RG, Bergé B, Fries W, Schmiedl A, Koroma S \& Hohenberger W 1998 Gastric fundectomy in the rat: effects on mineral bone metabolism, with emphasis on the gastrin-calcitonin-parathyroid hormone-vitamin D axis. Calcified Tissue International 63 433-441.

Sato M, Zeng GQ \& Turner CH 1997 Biosynthetic human parathyroid hormone (1-34) effects on bone quality in aged ovariectomized rats. Endocrinology 138 4330-4337.

Seedor JG, Quartuccio HA \& Thompson DD 1991 The bisphosphonate alendronate (MK-217) inhibits bone loss due to ovariectomy in rats. Journal of Bone and Mineral Research 6 339-346. 
Shen V, Dempster DW, Birchman R, Xu R \& Lindsay R 1993 Loss of cancellous bone mass and connectivity in ovariectomized rats can be restored by combined treatment with parathyroid hormone and estradiol. Journal of Clinical Investigation 91 2479-2487.

Shen V, Birchman R, Xu R, Otter M, Wu D, Lindsay R \& Dempster DW 1995 Effects of reciprocal treatment with estrogen and estrogen plus parathyroid hormone on bone structure and strength in ovariectomized rats. Journal of Clinical Investigation 96 2331-2338.

Stadil F \& Refeld JF 1973 Determination of gastrin in serum. An evaluation of the reliability of a radioimmunoassay. Scandinavian Journal of Gastroenterology 8 101-112.

Stenström M, Olander B, Lehto-Axtelius D, Madsen JE, Nordsletten L \& Carlsson GA 2000 Bone mineral density and bone structure parameters as predictors of bone strength: an analysis using computerized microtomography and gastrectomy-induced osteopaenia in the rat. Journal of Biomechanics 33 289-297.

Surve VV, Andersson N, Alatalo S, Lehto-Axtelius D, Halleen J, Väänänen K \& Håkanson R 2001a Does combined gastrectomy and ovariectomy induce greater osteopaenia in young female rats than gastrectomy alone? Calcified Tissue International 69 274-280.

Surve VV, Andersson N, Lehto-Axtelius D \& Håkanson R $2001 b$ Comparison of osteopaenia after gastrectomy, ovariectomy and prenisolone treatment in the young female rat. Acta Orthopaedica Scandinavica 72 525-532.

Surve VV, Höglund P \& Håkanson R 2002 Gastrectomized rats respond with exaggerated hypercalcemia to oral and intravenous calcium loads because of impaired ability of bone to take up $\mathrm{Ca}^{2+}$. Scandinavian Journal of Gastroenterology 37 523-530.

Thompson DD, Simmons HA, Pirie CM \& Ke HZ 1995 FDA Guidelines and animal models for osteoporosis. Bone 17 125S-133S.

Tovey FI, Hall ML, Ell PJ \& Hobsley M 1991 Postgastrectomy osteoporosis. British Journal of Surgery 78 1335-1337.
Tovey FI, Hall ML, Ell PJ \& Hobsley M 1992 A review of postgastrectomy bone disease. Journal of Gastroenterology and Hepatology 7 639-645.

Turner RT, Vandersteenhoven JJ \& Bell N 1987 The effects of ovariectomy and $17 \beta$-estradiol on cortical bone histomorphometry in growing mice. Journal of Bone and Mineral Research 2 115-122.

Uzawa T, Hori M, Ejiri S \& Ozawa H 1995 Comparison of the effects of intermittent and continuous administration of human parathyroid hormone(1-34) on rat bone. Bone 16 477-484.

Windahl SH, Vidal O, Andersson G, Gustafsson JA \& Ohlsson C 1999 Increased cortical bone mineral content but unchanged trabecular bone mineral density in female ER $\beta-/-$ mice. Journal of Clinical Investigation 104 895-901.

Woitge HW \& Seibel MJ 2001 Biochemical markers to survey bone turnover. Rheumatic Disease Clinics of North America 27 49-80.

Wojtyczka A, Bergé B, Rümenapf G, Schwille PO, Ballanti P, Schreiber M, Fries W \& Hohenberger W 1998 Gastrectomy osteopaenia in the rat: the role of vitamin B12 deficiency and the type of reconstruction of the digestive tract. Clinical Science 95 735-744.

Wronski TJ, Lowry PL, Walsh CC \& Ignaszewski LA 1985 Skeletal alterations in ovariectomized rats. Calcified Tissue International 37 324-328.

Wronski TJ, Cintron M, Doherty AL \& Dann LM 1988 Estrogen treatment prevents osteopaenia and depresses bone turnover in ovariectomized rats. Endocrinology 123 681-686.

Yamazaki I \& Yamaguchi H 1989 Characteristics of an ovariectomized osteopenic rat model. Journal of Bone and Mineral Research 4 13-22.

Received in final form 7 August 2002

Accepted 9 August 2002 